\title{
The Balloon Experimental Twin Telescope for infrared interferometry (BETTII): first flight
}

S. A. Rinehart, A. Dhabal, D. Fixsen, R. JuanolaParramon, D. Leisawitz, et al.

S. A. Rinehart, A. Dhabal, D. Fixsen, R. Juanola-Parramon, D. Leisawitz, S. Maher, J. E. Mentzell, L. Mundy, M. Rizzo, H. Sampler, E. Sharp, R. Silverberg, M. Casalprim Torres, T. Veach, J. Vila Hernández de Lorenzo, P. A. R. Ade, C. Tucker, E. Pascale, G. Savini, "The Balloon Experimental Twin Telescope for infrared interferometry (BETTII): first flight," Proc. SPIE 10700, Ground-based and Airborne Telescopes VII, 107000F (6 July 2018); doi: $10.1117 / 12.2311497$

Event: SPIE Astronomical Telescopes + Instrumentation, 2018, Austin, Texas, United States 


\title{
The Balloon Experimental Twin Telescope for Infrared Interferometry (BETTII): First Flight
}

S. A. Rinehart ${ }^{* a}$, A. Dhabal ${ }^{b}$, D. Fixsen ${ }^{b}$, R. Juanola-Parramon ${ }^{\text {ac }}$, D. Leisawitz ${ }^{a}$, S. Maher ${ }^{\text {ad }}$, J. E. Mentzell $^{\mathrm{a}}$, L. Mundy ${ }^{\mathrm{b}}$, M. Rizzo ${ }^{\mathrm{ac}}$, H. Sampler ${ }^{\mathrm{a}}$, E. Sharp ${ }^{\mathrm{ae}}$, R. Silverberg ${ }^{\text {af }}$, M. Casalprim Torres ${ }^{\mathrm{g}}$, T. Veach $^{\mathrm{h}}$, J. Vila Hernández de Lorenzo ${ }^{\text {ai }}$, P. A. R. Ade ${ }^{\mathrm{j}}$, C. Tucker ${ }^{\mathrm{j}}$, E. Pascale ${ }^{\mathrm{k}}$, G. Savini ${ }^{1}$

aNASA's Goddard Space Flight Center; 'University of Maryland - College Park, 'NASA Postdoctoral Fellow, dSSAI, ${ }^{\mathrm{e}}$ Alora S\&D, fSURA, gSUPAERO, hSouthwest Research Institute, ${ }^{\mathrm{i} C}$ atholic University, ${ }^{\mathrm{j} C a r d i f f}$ University, ${ }^{\mathrm{k}} \mathrm{La}$ Sapienza, ${ }^{1}$ University College London

\begin{abstract}
The Balloon Experimental Twin Telescope for Infrared Interferometry (BETTII) is an 8-meter far-infrared (30-100 $\mu \mathrm{m})$ double-Fourier Michelson interferometer designed to fly on a high altitude scientific balloon. The project began in 2011, and the payload was declared ready for flight in September 2016. Due to bad weather, the first flight was postponed until June 2017; BETTII was successfully launched on June 8, 2017 for an engineering flight. Over the course of the onenight flight, BETTII acquired a large amount of technical data that we are using to characterize the payload.

Unfortunately, the flight ended with an anomaly that resulted in destruction of the payload. In this paper, we will discuss the path to BETTII flight, the results of the first flight, and some of the plans for the future.
\end{abstract}

Keywords: Balloon, Far-Infrared, Interferometers, Star Formation

\section{INTRODUCTION}

The Balloon Experimental Twin Telescope for Infrared Interferometry (BETTII) was conceived as a tool for obtaining spectroscopic information in the far-infrared (30-100 mm) of closely-spaced sources. By flying on a high-altitude balloon above 120,000 feet, BETTII operates above nearly the entire atmosphere, giving access to this wavelength regime. With an 8-meter interferometric baseline, it is capable of separating sources that would be otherwise confused with singleaperture telescopes; this, combined with the double-Fourier instrument, allows BETTII to provide spatially-resolved spectroscopy of individual sources in crowded regions. At the same time, BETTII serves as a technical demonstration for future space-based interferometric missions.

BETTII was selected for funding through the ROSES/APRA program in 2010, and development begin in 2011 at NASA's Goddard Space Flight Center (GSFC). Over the next five and a half years, the payload was designed, constructed, and tested, and in 2016 was deployed to Fort Sumner, New Mexico for its Engineering Flight. The goals of this flight were to debug and calibrate the payload, to understand the performance of individual subsystems, and to assess operational optimization strategies. Unfortunately, due to poor weather, BETTII did not fly in 2016, and was rescheduled to the June 2017 campaign in Palestine, TX. On June 8, 2017, BETTII successfully launched from the Columbia Scientific Balloon Facility (CSBF) in Palestine.

BETTII's engineering flight was very successful, and based upon flight results, the team was confident that BETTII would be ready for its first Science Flight in 2018. Unfortunately, the flight ended with an anomaly that resulted in the near-total loss of the payload.

\subsection{Scientific Rationale}

The absence of high angular resolutions in the far-infrared leave us unable to answer a number of important scientific questions. BETTII is designed to address this lack. With an 8-meter fixed interferometric baseline, it is designed not to image sources, but rather to provide spatially resolved spectroscopy. The angular resolution provided by the spatial

* Stephen.A.Rinehart@nasa.gov; phone 1-301-286-4591 
interferometer, in combination with the double-Fourier instrument, allows the reconstruction of spectra of multiple sources within crowded fields, with the ability to distinguish individual sources with separations of $<1$ arcsecond. This has potential application across a wide range of astrophysics, but for the BETTII science flights, we are focusing on the formation of stars in clusters and in multiple systems.

Star formation is a fundamental process within the universe. At large scales, it determines the appearance and evolution of galaxies. At small scales, it leads to the formation of new planetary systems. And yet, there remain many unanswered questions about the physical processes involved, including those that lead to the formation of binary and multiple star systems (the majority of stars form in such groups). On scales between 15 and 10,000 AU, approximately $70 \%$ of all protostars exist in binary/multiple systems ${ }^{1,2}$, as observed through interferometric observations at sub/millimeter wavelengths (Figure 1). However, these observations are only sensitive to the emission from the circumstellar material, and do not effectively probe the characteristics of the individual protostars. NIR observations provide complementary information on nebulosity from scattered light and shock-heated molecular line emission. But, observations in the 5-200 $\mu \mathrm{m}$ range are also needed; at these wavelengths, the light can penetrate the obscuring material, and it is possible to sample the peak emission of the protostellar cores.

Unfortunately, observations in most of this wavelength range are impossible from the ground, and space-borne observations are plagued by confusion in these crowded region because of their inherently low angular resolution. The spatially-resolved spectroscopy provided by BETTII is designed to directly measure the individual Spectral Energy Distributions (SEDs) of protostars forming in binary/multiple systems or in clusters. This will be unique data, complementary to existing ground- and space-based data, with significant scientific potential. Using BETTII, we hope to obtain new insights into the formation and coeveolution of stars in clusters, potentially answering long-standing questions about the physical processes involved.

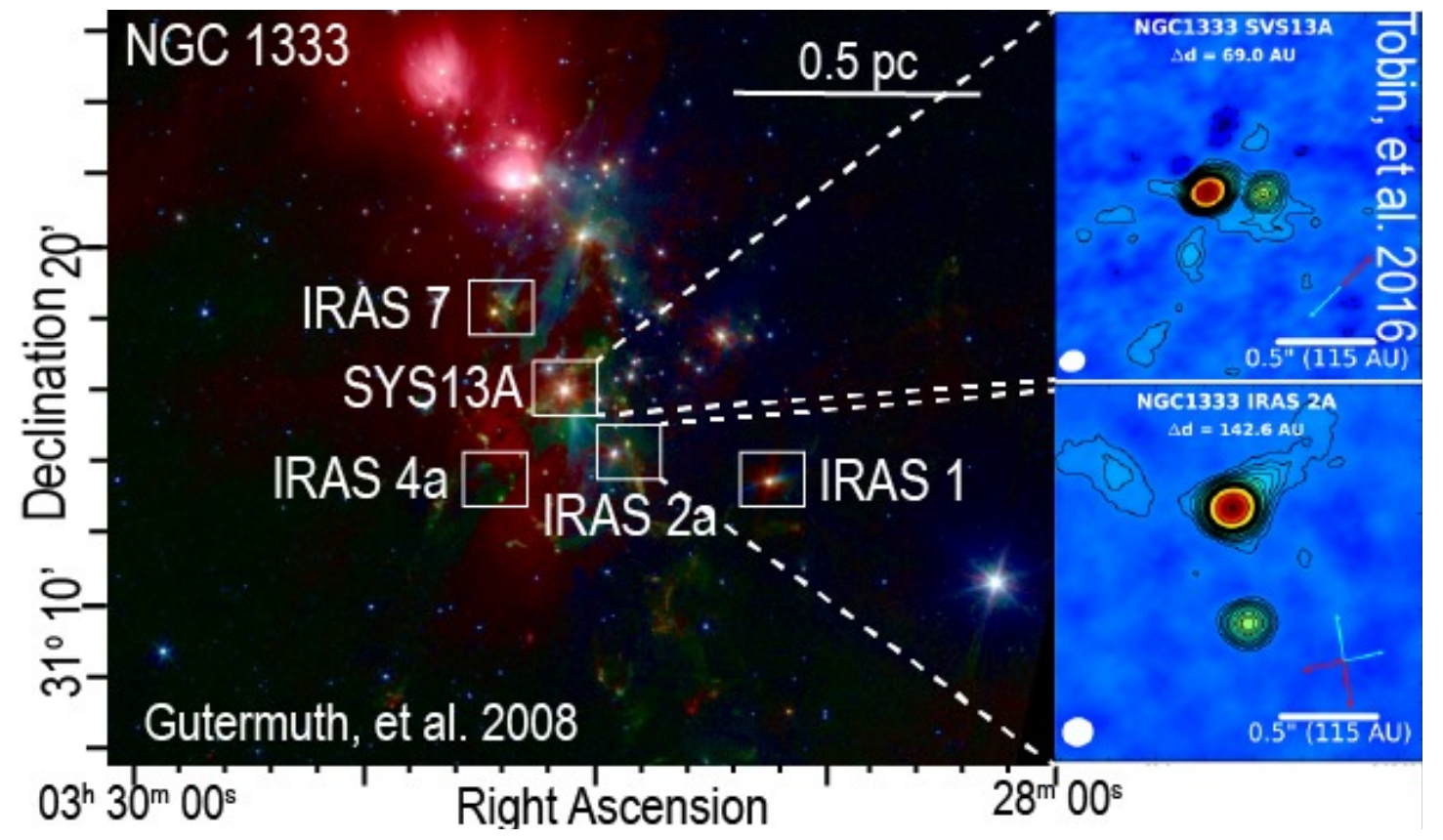

Figure 1: NIR observations of young star-forming clusters ${ }^{3}$ reveal rich structure in star-forming regions, and interferometric sub/millimeter observations reveal multiplicity in many of these "individual" sources ${ }^{1}$. BETTII will bridge the gap between these observations, allowing us to measure the mass accretion rates for individual protostars and providing new insights into the Initial Mass Function (IMF).

\subsection{Technical Importance}

The potential value of interferometry for probing fine angular scales has been known for many years. The 2000 Decadal Report ${ }^{4}$ argued for the importance of developing space-based interferometers, suggesting that development of a farinfrared interferometry mission could occur in the 2010s. Of course, the 2000 Decadal was extremely ambitious, and much of their vision did not come to fruition, but the core arguments for developing space-based interferometers remain - 
interferometry is the only practical method for obtaining data at the fine angular scales needed to address fundamental astrophysical questions across a wide range of wavelengths and scientific topics (see Table 1 for a few examples).

The underlying arguments for interferometers were recognized once again in the 2013 NASA Astrophysics Roadmap, "Enduring Quests, Daring Visions", "All notional missions in the Visionary Era are interferometers, and technology maturation of interferometric techniques is thus highly relevant to realizing the science vision." (emphasis in both quotes is from the report). The Roadmap specifically identifies the need for interferometers operating across all wavelengths, for science ranging from detailed studies of exoplanets to understanding the physics in the accretion disks of black holes. It also notes "Interferometry has historically progressed from longer wavelengths, where technological challenges are less extreme, to shorter wavelengths... FIR interferometry may again be a logical starting point that provides a useful training ground while delivering crucial science." BETTII is the first step on this path, as NASA's first long-baseline stellar interferometer in "space." It will demonstrate scientific capabilities of and technologies/techniques needed for future space interferometers.

\section{TAKING BETTII TO FLIGHT}

\subsection{The BETTII Design}

Over the course of the first five years of the Project, the team designed, built, and tested BETTII. The overall design of BETTII is described in detail in Rinehart et al. $(2014)^{6}$ and elsewhere, ${ }^{7,8}$ BETTII is a complex system, but for simplicity, can be considered as the combination of three major subsystems: the external optics; the instrument; and the exoskeleton and control system.

External Optics: The external optical system is designed to collect two 50-cm diameter wavefront samples and to provide $2.5-\mathrm{cm}$ diameter, co-phased, commonly rotated beams to the central double-Fourier beam-combining instrument ${ }^{9,10}$. A raytrace of this system is shown in Figure 3. Siderostats (mounted on rotation stages) collect the beams and transfer them to four-element telescope assemblies to provide the compression ${ }^{11}$. Co-phasing is provided by a warm delay line (WDL) in one interferometric arm, while common rotation is provided by a K-mirror assembly (KMA) in the other arm. Both the WDL and the KMA include a fast tip/tilt mechanism for fine pointing control. All optical elements (including the instrument/cryostat) and control system sensors (e.g. star cameras) are mounted to the metering truss (Figure 2), constructed of carbon-fiber and steel. This truss was designed to be light $(<100 \mathrm{~kg})$, strong (able to withstand 10-g loads),

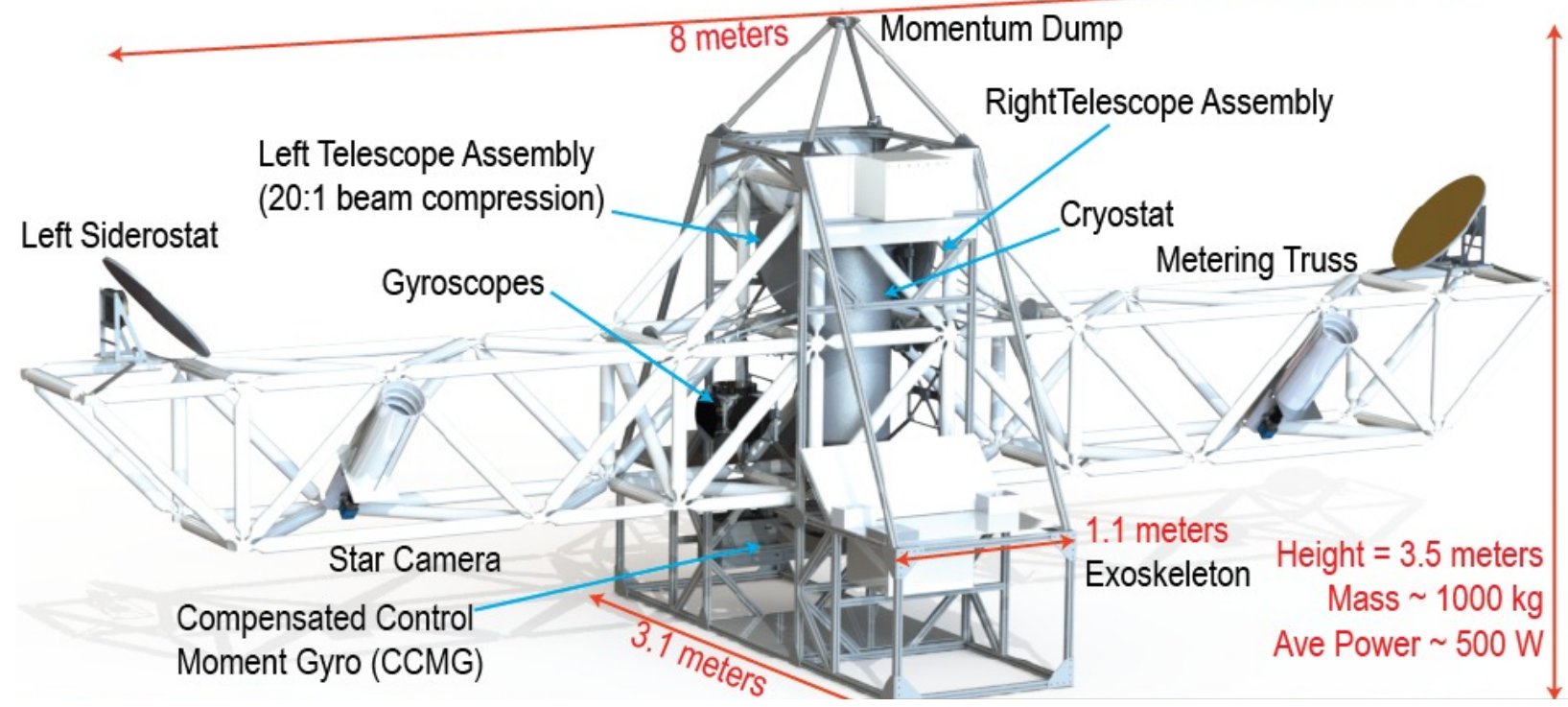

Figure 2: This render of the BETTII payload shows the critical features of the experiment. The siderostats are mounted at the ends of the 8-meter truss, which also holds all other optical elements, including the cryostat. Inside the cryostat is the doubleFourier instrument. Electronics and the Compensated Control Moment Gyro are mounted to the exoskeleton, connected to the metering truss through passive vibration dampers. 


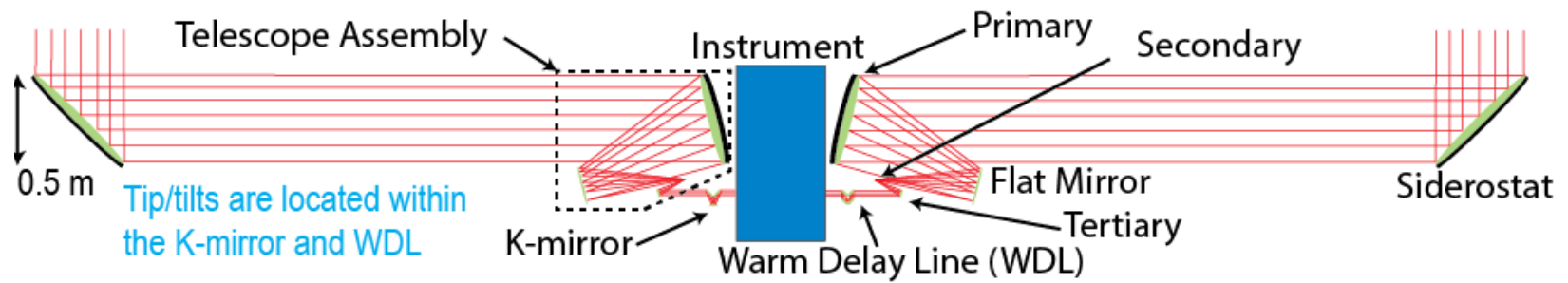

Figure 3: The design of BETTII's warm optics is shown here. Siderostats bring light to the pair of telescope assemblies that provide a 20:1 collimated compressed beam. The warm delay line on one side co-phases the two arms, while the K-mirror assembly provides corotation.

and stiff (with vibration modes frequencies $>25 \mathrm{~Hz}$ ); lab testing and flight data show that our design exceeded all of these requirements. The metering truss itself is mounted within the gondola structure through vibration isolators.

Instrument: The instrument is defined, in this context, as the cryostat and all components within $\mathrm{it}^{12}$. Light enters the dewar through a thin polypropylene window. Inside the cryostat, there is a "cool' volume (LN2-cooled) for the nearinfrared tracking channel, consisting of optics and an H4RG detector array, and a "cold" volume $\left({ }^{4} \mathrm{He}-\mathrm{cooled}\right)$ for the science channel, consisting of optics (Figure 4), quasi-optics, the cold delay line (CDL), four transition edge sensor (TES) bolometer arrays and associated cold electronics, and the ${ }^{3} \mathrm{He}+{ }^{4} \mathrm{He}$ sorption refrigerator (from Chase Research) for cooling the detectors to $300 \mathrm{mK}$.

The CDL was designed using heritage from other Goddard-developed mechanisms ${ }^{13,14}$, and was successfully tested both in the lab and in flight. During normal operation, the CDL carries out a scan every 3 seconds. Quasi-optics include blocking filters, the beam combiner, and FIR dichroics, and were contributed to the project by Cardiff University. At the heart of the instrument are four $9 \times 9$ close-packed linear arrays of multiplexed superconducting transition edge sensor (TES) bolometers ${ }^{15}$ incorporating the Backshort Under Grid architecture ${ }^{16}$. The flight arrays were tested and characterized in the laboratory. These arrays are digitally controlled and read out using a single ambient-temperature Multi-Channel Electronics (MCE) unit developed by the University of British Columbia ${ }^{17}$.

The H4RG array is read out using a standard GENIII controller ${ }^{18,19}$. The controller configuration is standard, with a minor modification to use a PCIe interface card to increase data throughput to $512 \mathrm{MB} / \mathrm{s}$. Controller machine code was developed based upon software developed at GSFC for H2RG arrays but was modified for BETTII to allow for readout of multiple subarrays at rates of $>100 \mathrm{~Hz}$. This work was done in cooperation with the Wide-Field Infrared Survey Telescope (WFIRST) project, which also provided the array.

Exoskeleton and Control System: All electrical systems (e.g. flight computer, MCE) and non-optical control system actuators (e.g. the CCMG, the rotator) are mounted on the exoskeleton. The exoskeleton, constructed of aluminum framing, meets the same 10-g safety requirement as the metering truss, but has relaxed stiffness requirements, as the metering truss is attached via passive vibration dampers. All electrical systems are slaved to a single master clock to minimize system

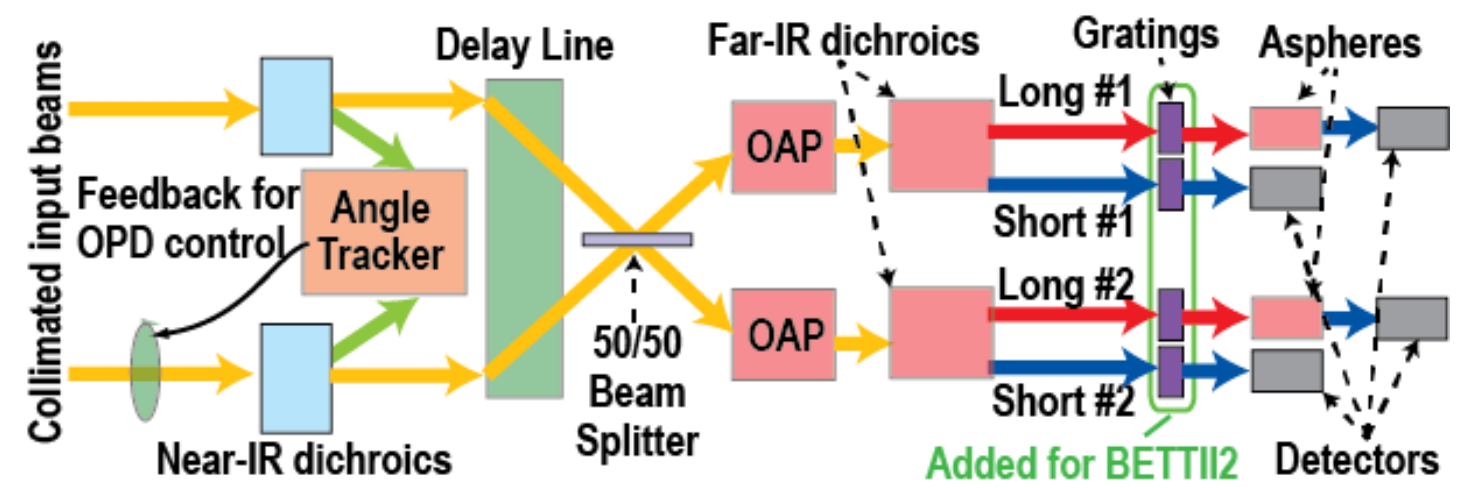

Figure 4: The BETTII optical system provides both spatial and spectral information simultaneously through double-Fourier operation, shown in block diagram form here; light is focused onto the arrays by off-axis parabolas (OAPS).. The gratings, circled on the right, were not present for the first BETTII flight but are being incorporated into the design for the next flight. 


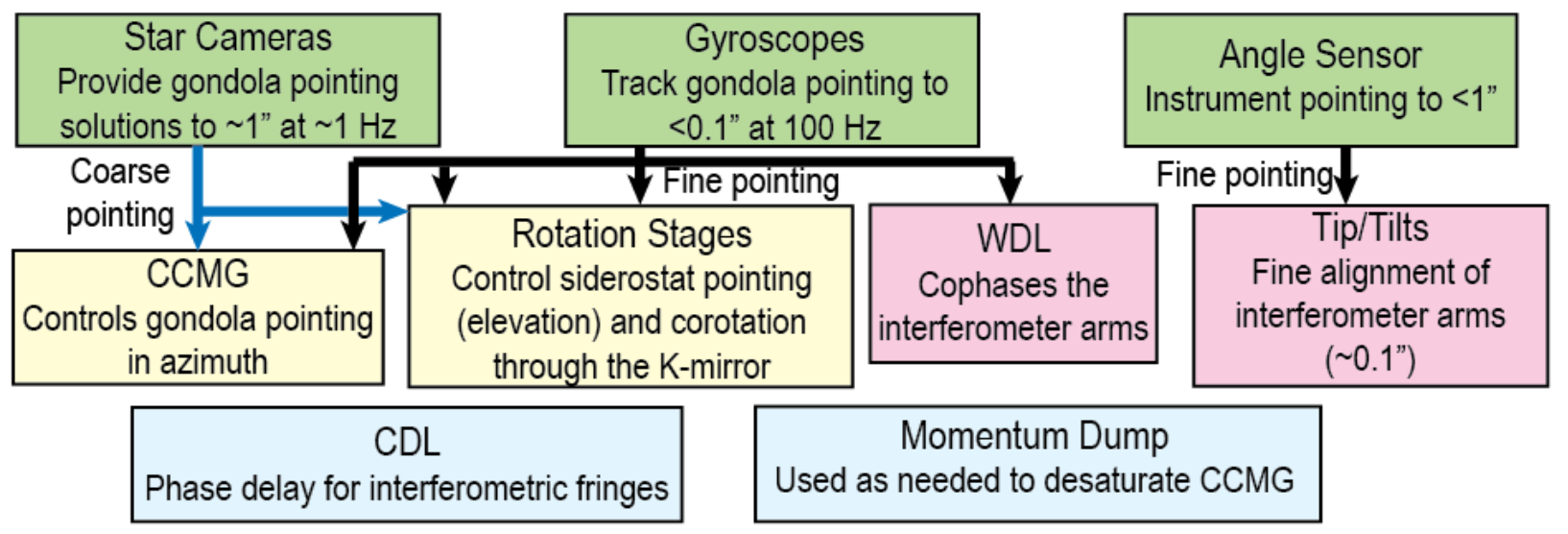

Figure 5: Control system sensors include a pair of star cameras, gyros, and an angle sensor; these provide the data needed for gondola slews, coarse pointing, and fine pointing. The control is provided by the CCMG, rotation stages, and tip/tilts. The data also allows for cophasing using the warm delay line and corotation using the K-mirror. The CDL provides optical path difference for interferometric fringes; the momentum dump is used as needed to prevent control system saturation.

noise, and additional ancillary sensors (e.g. thermometers) assist with post-flight performance assessment and data quality determination.

Sensors within the control system include two star cameras ${ }^{20}$, three fiber optic gyroscopes (OptoLink SRS-2000), and the angle sensor (the NIR channel of the instrument). Actuators include the Compensated Control Moment Gyros (CCMG), the momentum dump mechanism, rotation stages for the two siderostats and the K-mirror assembly, the warm delay line, two tip/tilt mechanisms, and the cold delay line (Figure 5).

The control system has four modes of operation ${ }^{9,21}$. These modes determine gondola pointing (lost mode), slew to a target (slew mode), lock-in on a target of interest (lock mode), and follow the target during data acquisition (tracking mode). In tracking mode, the actuators allow fine pointing control using the tip/tilts to $\sim 1^{\prime \prime}$, while the sensors provide knowledge to better than $0.5^{\prime \prime}$. Simulations show that with the inclusion of interferometric fringes from the science channel, we will be able to reconstruct pointing to better than $\sim 0.1 "$, exceeding pointing stability requirements.

\subsection{The Fort Sumner Campaign}

In 2016, construction of BETTII was completed, and it was deployed to Fort Sumner, TX for an Engineering Flight. Over the course of $\sim 1$ month in the laboratory there, and taking advantage of a mobile crane outside, system-level tests were carried out to maximize confidence in a high-quality flight. On September 27, 2016, BETTII rolled out to the launch line for an evening launch; this came after weeks of waiting for bad weather to clear. Such evening launches are a rarity in Fort Sumner, with nearly all launches occurring in the morning due to prevailing local weather conditions. An evening launch was ideal for BETTII as it would maximize the time available for science operation post-sunset; to carry out a minimum set of commissioning activities, BETTII needs $\sim 4$ hours of dark observing time, a challenging requirement for morning launches. Unfortunately, the low-level winds (at around 500 feet) did not die down as predicted, and the launch was scrubbed. Another attempt was possible for early the next morning, but simulations showed that this flight was likely to get less than two hours of dark time - failing to meet flight requirements for BETTII'. Weather forecasts showed no possibility of a launch/flight meeting BETTII requirements during the remainder of the campaign, so BETTII and the team returned to GSFC.

Despite the scrubbed flight attempt, the BETTII team gained valuable experience through the process of final integration, system testing, and incorporation of components from the Columbia Scientific Ballooning Facility. Based upon the lessons-learned, the team spent the next $\sim 6$ months making improvements to the payload prior to the next launch attempt out of Palestine, TX. Amongst the improvements made were: (1) upgraded flight software; (2) making a more robust power system; (3) adding optical alignment fiducials to the payload; (4) redesigning the cold electronics board for FIR

\footnotetext{
${ }^{\dagger}$ In fact, the payload that launched on the morning of Sept. 28, 2016, had less than 1 hour of dark time before the flight was terminated.
} 


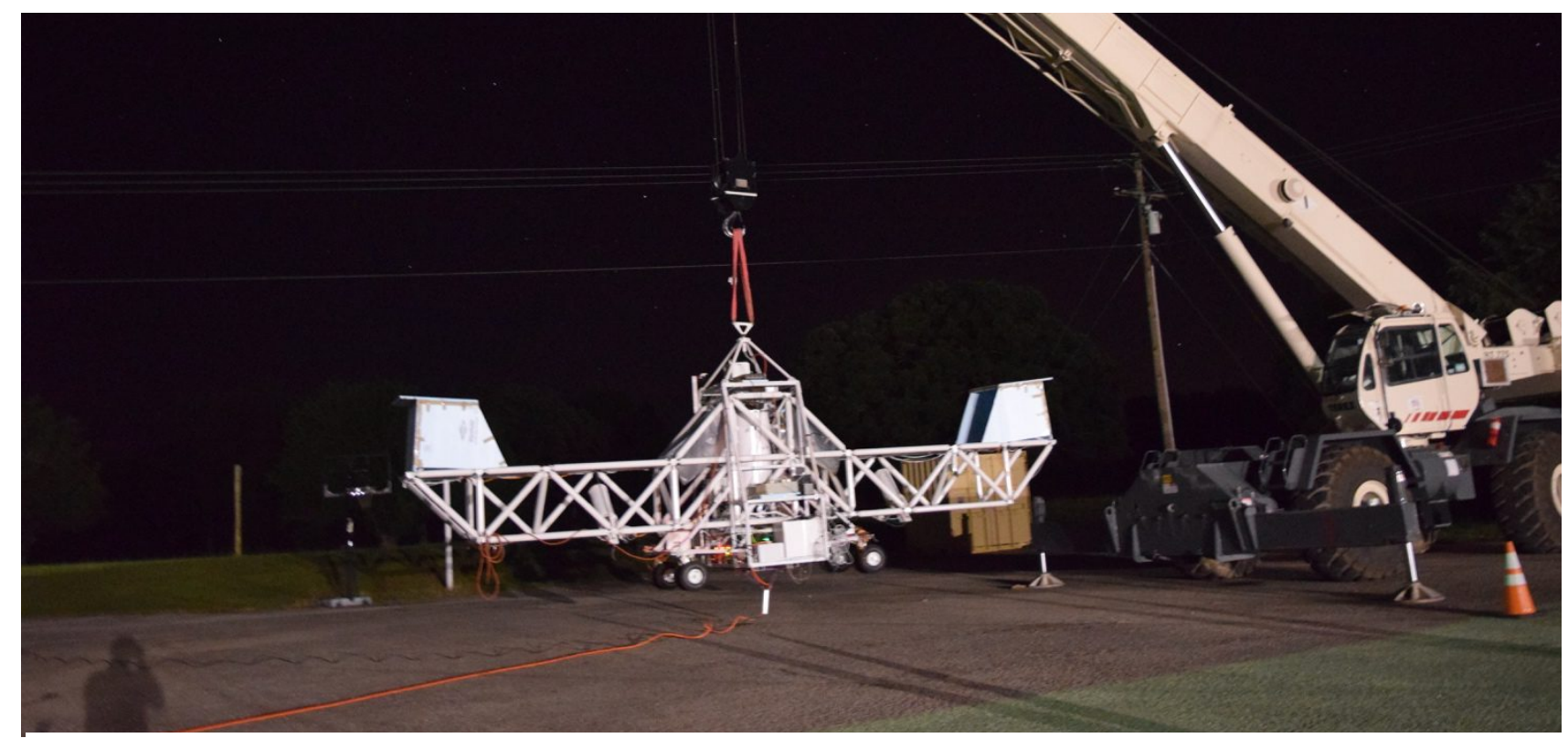

Figure 6: In both Fort Sumner and in Palestine, system-level tests of the payload were carried out both in the high bay facility and outside using a mobile crane.

detector readout; and (5) incorporating an H4RG detector array into the near-infrared system. With these improvements, BETTII was more capable, and ready for launch.

The most significant of these activities was the incorporation of the H4RG array. The Leach controller was modified, as was the software, in order to incorporate a BETTII flight mode that simultaneously operates two windows in sample up the ramp mode. In this mode, window readout rates are upwards of $100 \mathrm{~Hz}$, with overall speed of 300,000 pixels per second.

\subsection{The Palestine Campaign}

In May 2017, BETTII was shipped to Palestine, and again the BETTII team deployed to prepare it for flight. With the experience from the Fort Sumner campaign, preparations went smoothly (mostly) and BETTII was declared ready for flight on June 1, 2017. After several days waiting for good weather, BETTII launched from Palestine on its maiden Engineering flight on June 8, 2017.

\section{THE BETTII FLIGHT}

Prior to flight, a detailed plan was developed for the sequential activation of payload components. During ascent, as each component was turned on, diagnostics were performed to verify that they were active and were providing appropriate telemetry. This was aided by a sophisticated ground software package that provided the ability to simultaneously monitor nearly every system on the payload. By the time BETTII achieved float altitude, we were able to verify that all systems were on, and we began exercising all of the individual system components.

Over the course of the night, we exercised system components and had a number of significant successes. These included:

- Validation of the optical system, including retention of optical alignment through launch and stability of the metering truss.

- Demonstration of closed-loop pointing control, using the rotator. While the CCMG was not operational during flight (see below), the momentum dump mechanism was used to slew and brake the payload, achieving stable angular velocities of $\sim 20 \mathrm{arcsec} / \mathrm{sec}$. Overall rotator performance was $\sim 10 \mathrm{x}$ better than measured in the laboratory. More details on control system performance can be found in Vila et al. 2018 (these proceedings ${ }^{22}$ ).

- End-to-end functional tests of all flight electronics and software; all electrical systems survived the extreme cold of passage through tropopause. 
- Measure thermal performance; the only deviation from pre-flight predictions was that the nodes on the bottom of the truss remained $\sim 5^{\circ} \mathrm{C}$ warmer than the top of the truss (due to radiative coupling with the Earth). These deviations were symmetric across the payload.

- Successful validation of the H4RG near-infrared detector. Over 250,000 images were collected throughout the night, and this was the first operation of an H4RG in a flight environment. This data is currently being analyzed to characterize noise performance of the array and will be published in a future paper. We also demonstrated multi-windowing capability of the H4RG, something never before done on a flight instrument ${ }^{23}$.

Performance of the control system was particularly encouraging. The better-than-expected performance of the rotator helps provide a high level of confidence in the capability of the CCMG. The CCMG has been shown to meet requirements in the laboratory, and conservative simulations of on-flight performance incorporating flight data show that we should easily meet BETTII's pointing requirements.

Overall, $\sim 80 \%$ of BETTII's systems worked as expected; issues arose with four systems, as discussed below, and four systems could not be fully validated on flight (e.g. the ${ }^{3} \mathrm{He}$ fridge). With the excellent overall performance, and with a clear understanding of the issues that arose, BETTII was well-placed for a successful science flight.

It is also worth noting that during daylight hours, the optical alignment of the system appeared to hold, and that all other systems (with the exception of the star camera) appeared to perform nominally. Additional testing is needed to fully validate the design for daytime use; these tests can be made during future flights. This would open the door for longduration flights.

\subsection{Issues}

Four issues arose in the course of the BETTII flight; three of these were discovered during the flight (one of which is very minor), while the fourth (and most significant) was discovered at the end of the flight.

The first of the two significant in-flight issues was a cold leak in the cryostat. The leak had been found prior to launch, caused by a crack in the fill tube weld at the base of one of the helium fill tubes. We mitigated the problem by installing a Stycast plug; the additional thermal loading kept the fill tube temperature high enough to prevent the cold leak from opening, at the penalty of reducing cryostat hold time from $\sim 30$ hours to $\sim 14$ hours - still sufficient to meet requirements for an evening launch. During rollout, the leak reopened (likely due to "slosh" in the liquid helium tank); shortly after launch, we determined that the liquid helium reservoir was empty. This prevented an on-flight test of the ${ }^{3} \mathrm{He}$ fridge and full characterization of the FIR detector arrays, but both of these were thoroughly tested in the lab and there is no expectation that flight performance would be different. All other elements in the cryostat (e.g. the H4RG, the CDL) were successfully tested.

The second issue was with the control system. The Galil microcontroller used to control the CCMG reaction wheels successfully turned on, and diagnostics received through a serial interface indicated that it was functioning normally. However, it did not respond to commands issued through the primary communication port (an Ethernet line). We established that the cause of the problem was that the Galil lacked an Internet Protocol (IP) address; in the laboratory, it had automatically retrieved a dynamically assigned IP address on startup through the local wireless network ${ }^{\ddagger}$. On launch, however, the Galil was turned off (to avoid potential damage to the reaction wheels); when turned on during ascent, the lack of a network led to no IP assignment. This prevented us from spinning up the reaction wheels and limited our ability to point the gondola to coarse pointing using the momentum dump mechanism. With the momentum dump alone, we were able to achieve $\sim 20$ " pointing, and verify the accuracy of the control system sensors, but fine pointing was not possible.

The third issue (extremely minor) occurred with a GPS sensor mounted on BETTII. The unit unexpectedly stopped functioning at around 40,000 feet, instead of the 65,000 feet specified by the manufacturer. This was likely caused either by an error in the GPS flash memory or parsing of incorrect messages; we have data to isolate this problem, but given that the GPS provides ancillary data only, and only during ascent, this is not a priority.

* GSFC computer security rules restrict the use of static IP addresses, which led to our use of the dynamic protocol. 

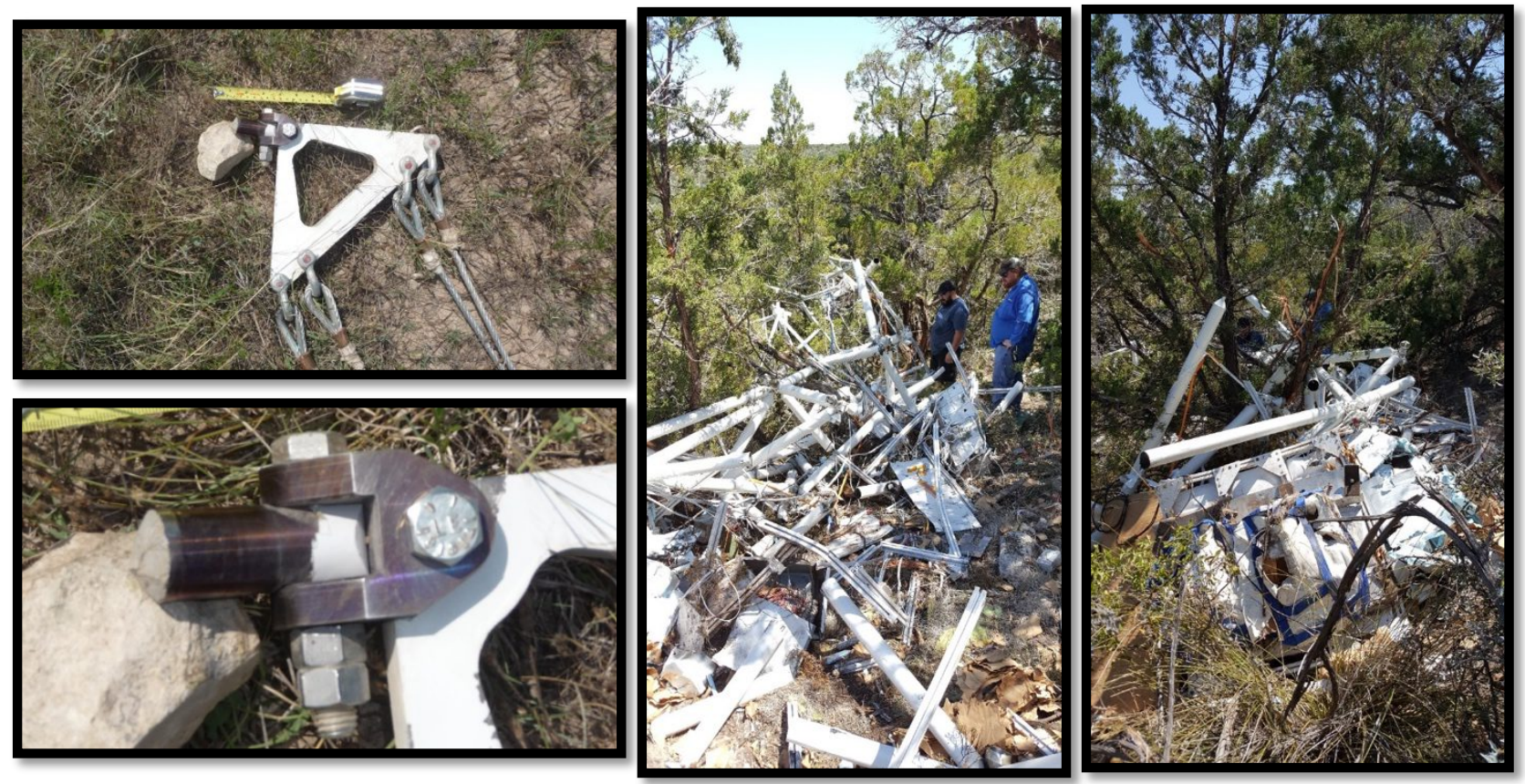

Figure 7: BETTII fell 135,000 feet in 12 minutes: it did not survive impact. The left two pictures show the truck plate attached to the parachute, along with the rotator pin. The right two pictures show the remnants of the payload.

\subsection{The Last Issue, and the End of Flight}

As an Engineering Flight, BETTII's maiden voyage was very successful; we were able to demonstrate the function of nearly every system, and real-time analysis of telemetered data showed excellent performance overall. The two major issues were arose during flight were rapidly diagnosed and had clear paths to resolution. Unfortunately, the most significant issue arose after the payload was turned off, when BETTII was released from the balloon. At this time, an anomaly occurred, resulting in the separation of the payload from the parachute. BETTII fell 136,000 feet in 12 minutes, and the loss was near-total. A NASA investigation post-flight showed that this problem occurred through an error cascade: the steel pin that attached the gondola to the payload had snapped, due to a poor material choice (the steel used was very strong but is brittle at cold temperatures). The BETTII team's review failed to identify the problem, as did the CSBF safety review. Fortunately, aside from the payload (and one tree), nothing was harmed upon BETTII's landing. In addition, of the ten solid state drives on the payload, eight were recovered. Data was stored in mirrored solid state drives, and all (over a terabyte) of the flight data was recovered (including H4RG images, extensive engineering and monitoring data, etc.).

\section{POST-FLIGHT ACTIVITIES}

Shortly after the "landing", NASA calssified the event as a "close call". A team from the CSBF, with the assistance of a member of the BETTII team (T. Veach) began the recovery effort. To support the subsequent investigation, the landing site (Figure 7) was thoroughly documented prior to debris removal. The remnants themselves were removed from the site by helicopter and loaded into a truck for transport back to Palestine. These parts were sequestered upon arrival, pending investigation. At the same time, members of the BETTII team worked with personnel from the Balloon Program Office to identify lab equipment and documentation that would support the investigation; relevant documentation was also sequestered. All other materials were packed up and shipped back to GSFC. Lessons learned from the recovery are discussed in another paper in this conference ${ }^{24}$.

\subsection{Triage}

One of the first components of BETTII returned to GSFC were the solid state drives: as mentioned above, 9 of the 11 drives survived the landing. In coordination with the investigators, the BETTII team began examining the data, both to understand the on-flight performance and to look for any information that might shed light on the anomaly. As the investigation proceeded, the team was given permission to return to Palestine to examine the remnants of the payload and 

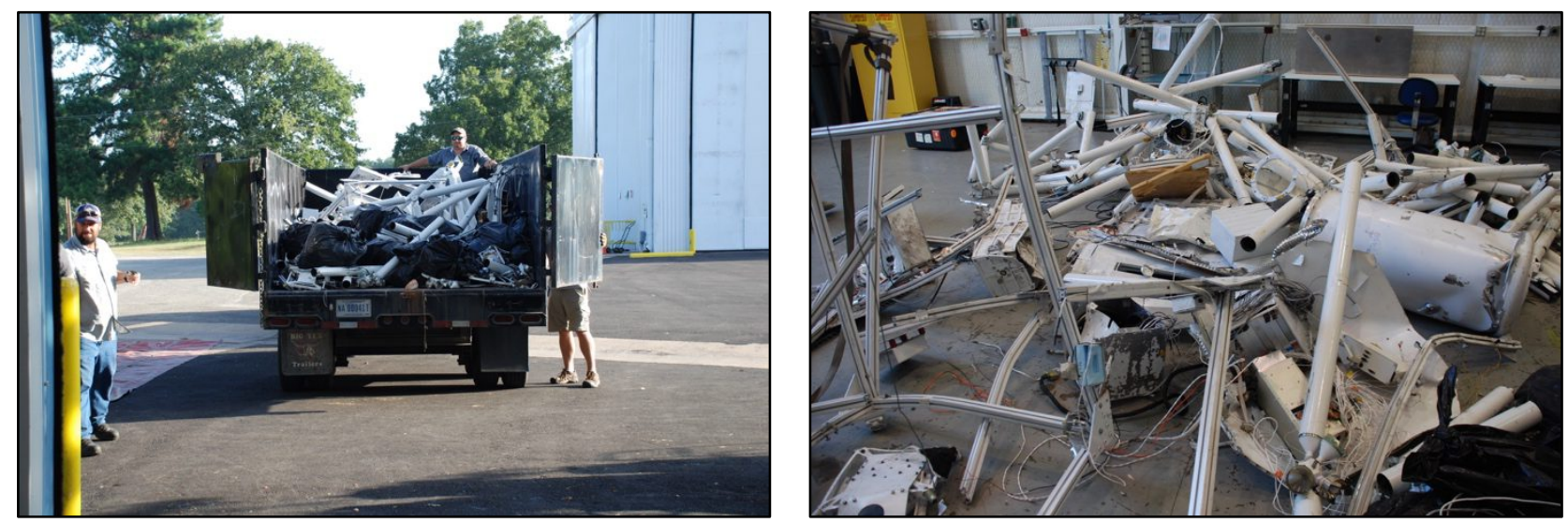

Figure 8: The remains of BETTII were delivered back to the CSBF facility in Palestine in a dump truck (left): some of the pieces on the high bay floor are shown at right.

to determine which components were potentially salvageable (Figure 8). Over the course of two days, three members of the BETTII team sifted through the debris. All structural elements were discarded; while some appeared intact, the likelihood of microscopic fracturing makes them unsuitable for reuse. A number of electronics components were recovered, if there was no or little visible damage. This included a number of connectors, electronics boards (including all of the cards from the MCE), and two of the three gyros. All four of the large external mirrors were recovered; while all showed small defects, only one of the siderostats showed significant damage; all four of these were returned to GSFC. The majority of small mirrors showed no visible damage and were also returned.

The cryostat itself was clearly damaged beyond recovery; it was eight inches shorter following landing, as the bottom of the dewar had "accordioned". To determine whether internal components had survived, we opened the cryostat (using a circular saw). We were able to remove the majority of the instrument components, including the entire optical bench. The $\mathrm{He}^{3}$ fridge, the $\mathrm{CDL}$, and the detector arrays were all lost, but the majority of optics (including the quasi-optics) were intact.

Since returning these components to GSFC, we have been able to verify that all of the MCE cards are fully functional. The large mirrors have been transported back to the Precision Engineering Center at North Carolina State University, where they were originally manufactured, for more detailed examination. Further testing of components, however, has not been possible due to limited resources.
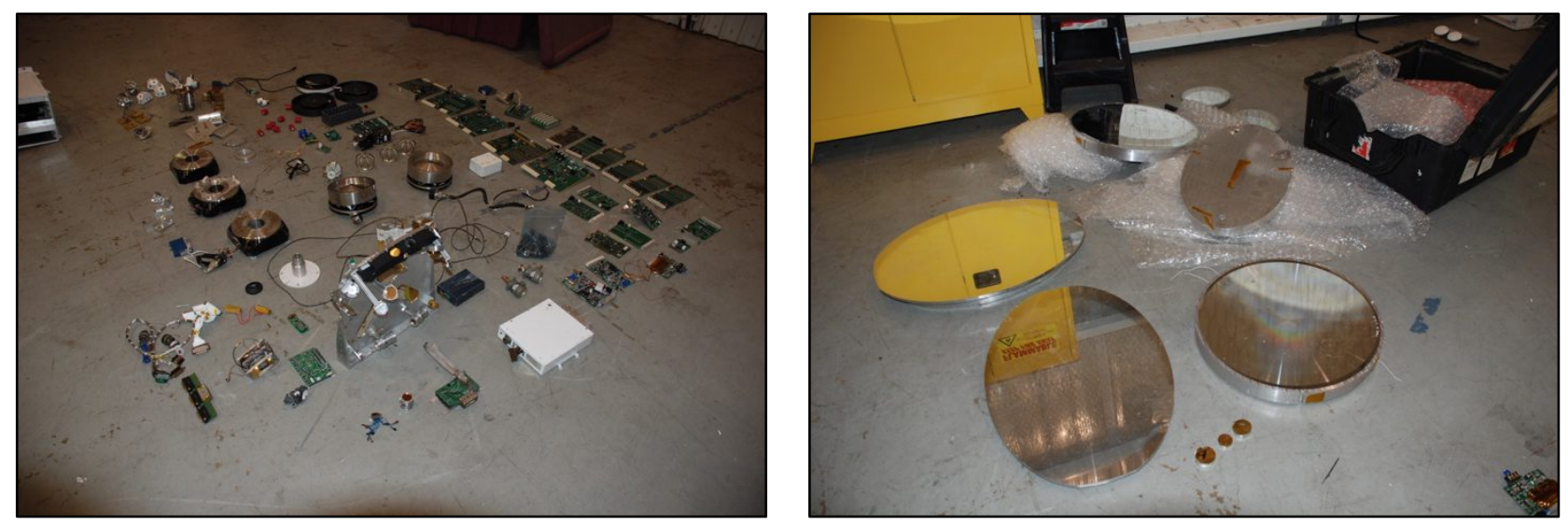

Figure 9: Triage activities allowed us to identify a number of components that were potentially reusable, including a large number of electrical components and the large mirrors. 


\section{THE PATH FORWARD}

Following the flight, the team developed a plan forward to return to flight as BETTII2, to complete the scientific and technical objectives of BETTII. This plan reuses the BETTII design, incorporating a few changes (discussed below) to improve performance, reduce risk, and/or reduce cost. Through the combination of design reuse, the already-complete flight and ground software, and the salvaged hardware, the cost of building BETTII2 is dramatically reduced - less one third the cost of developing BETTII. However, NASA HQ made a programmatic decision to curtail funding for the BETTII project, providing resources only to partially analyze flight data. Despite this setback, with generous internal support from GSFC, the team has begun developing BETTII2, with the hope of returning to flight as early as 2020.

\subsection{Our Starting Point}

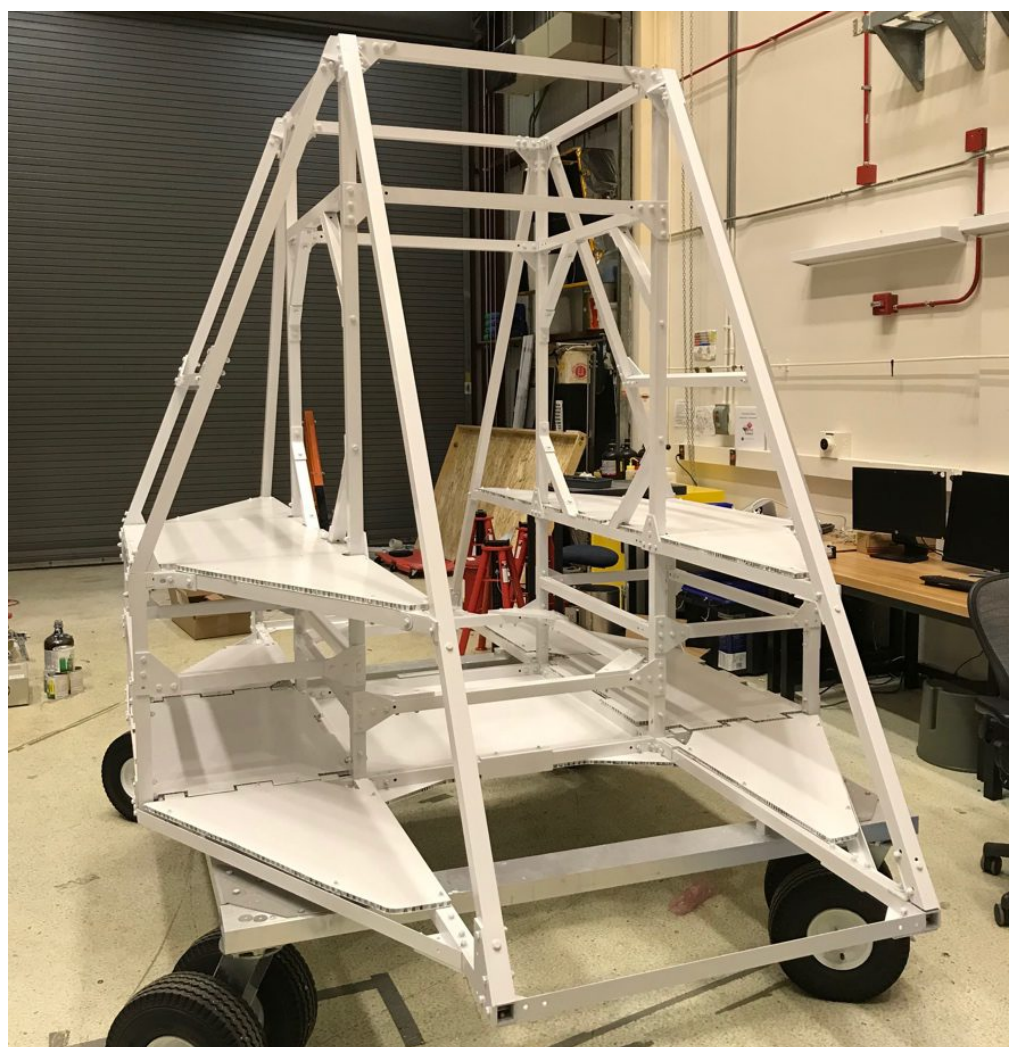

Figure 10: The exoskeleton for BETTII2 is already complete, as is the new power system. These are particularly valuable steps, as they will simplify the integration and testing of all subsystems as they become available.

Fortunately, the rebuild of BETTII2 has a significant start already. The recovered components are a significant part of this, but BETTII2 also benefits from a number of flight spares. The most significant of these are new FIR arrays. While the original flight arrays were lost, the original detector lot yielded significantly more arrays than the four that were needed. Four of these spares have now been completed and are ready for flight. With new cold electronics boards, in development now, these detectors will be ready for BETTII2. Other significant flight spares include the flight computer and components for the CCMG. The CCMG is being rebuilt now, using these components and internal funding.

GSFC support has also allowed us to purchase several key components needed for BETTII2, including a new cRio FPGA (BETTII's "brains"), a new gyro, and a helium-3 fridge. It has also allowed us to begin building the infrastructure for BETTII2. The new exoskeleton has been completed (Figure 10); while similar to the BETTII design, it is modified to incorporate aluminum tubing and larger panels for mounting subsystems. This design has been thoroughly modeled and significantly exceeds flight requirements. We have also designed and built a new power system. This new power system uses a single large battery pack to power a set of isolating Vicor DC-DC converters. This system provides stable, very low noise power for the entire payload, and helps mitigate potential ground-loop issues. 
BETTII2 will also benefit from reuse of other time-consuming and costly elements from BETTII, including all of the flight software, optical alignment procedures, and all of the necessary laboratory and ground system equipment.

\subsection{Improvements for BETTII2}

In addition to the improvements in the exoskeleton design and power train, described above, BETTII2 will include several other upgrades based upon the lessons-learned from the Palestine campaign. The metering truss will be constructed of 2" carbon fiber tubes with titanium fixtures (rather than 3" tubes and steel fixtures); this reduces overall mass, increases stiffness, reduces thermal distortion, and meets strength requirements, while also resulting in a $\sim 15 \%$ cost savings. We will also replace our in-house star camera design with a copy of the star camera used by the SuperBIT project (the lenses used for our design are no longer available). Their camera is compatible with our software, meets all requirements, and can be built more cheaply than our previous design. BETTII2 will also provide improved synchronization of electronics components, universalizing the one-clock architecture across the payload (e.g. incorporating this into the H4RG readout, CCMG operation, etc.).

We have also redesigned the instrument cryostat. The new design incorporates several features of the BETTII cryostat, providing a hold time of $>30$ hours and maintaining optical alignment independent of temperature. However, unlike the old dewar, simplification will facilitate work on the instrument by allowing independent access to each the optical and cryogenic systems. It also allows future integration of a cryocooler, needed for long-duration flights. The simpler design also reduces cost relative to the BETTII dewar.

The most significant design change, however, is the incorporation of a dispersed back-end to the instrument. Prior to BETTII's first flight, the team had been considering this for a future upgrade; the building of BETTII2 provides an opportunity for its inclusion. The instrument optical design remains largely the same, but includes a grating in each of the FIR science channels. The division of the waveband into multiple spectral channels reduces the background flux for each while also increasing the width of the interferometric fringe packets (i.e. the number of bright fringes increases). This improves sensitivity by the inverse root of the bandwidth ${ }^{25}$; using the 9-pixel width of the array, sensitivity improves by a factor of 3 over the full $30-100 \mu \mathrm{m}$ spectral band, at the cost of reducing the overall field-of-view. Additional sensitivity improvement is possible by increasing dispersion and reducing the band coverage. The addition of a grating is low-risk; both manufacture and incorporation of gratings is straightforward, and this technique has long history in ophthalmological imaging $^{26}$. Details of this new design can be found in Dhabal et al. (these proceedings) ${ }^{27}$.

The final significant design change is to the rotator. The new pin design uses titanium (which is ductile even at low temperatures) and incorporates other changes to address the failure from the BETTII flight. Further, the inclusion of a new clutch system will reduce shock to the pin on payload release. This design has been analyzed and shown to meet requirements; it is currently being validated by an independent engineering group at GSFC. Following validation, we will build multiple pins, including enough for proof testing and analysis.

\section{SUMMARY}

Ultimately, the BETTII flight was very successful. Of the three major issues (the cold leak, CCMG communications, and the rotator failure), all three are well-understood and have been addressed in planning for BETTII2. Significant progress has already been made towards the reflight, and with continued support, BETTII2 could be ready for flight as early as 2020. The first flight will focus on Recommissioning and will again focus on the testing and characterization of the payload needed to pave the way for future science flights. The tools needed for analysis of flight data are in-hand and will allow us to quickly assess performance on-flight, allowing for real-time optimization of some system elements. Data analysis tools are also complete and have been tested using simulated data; only minor modifications to this software will be necessary for working with the dispersed interferometric data. BETTII2 will complete the goals set out for BETTII, providing unique new scientific data for studying star formation while also demonstrating the fundamental techniques needed for space-based interferometric missions of the future. 


\section{REFERENCES}

[1] Tobin, J., Looney, L, Li, Z-Y., Chandler, C., Dunham, M., Segura-Cox, D., Sadavoy, S., Melis, C., Harris, R., Kratter, K., and Perez, L., "The VLA Nascent Disk and Multiplicity Survey of Perseus Protostars (VANDAM). II. Multiplicity of Protostars in the Perseus Molecular Cloud", ApJ, 818, 73 (2016).

[2] Chen, X., Arce, H., Zhang, Q., Bourke, T., Launhardt, R., Jørgensen, J., Len, C.-F, Foster, J., Dunham, M., Pineda, J., and Henning, T., "SMA Observations of Class 0 Protostars: A High Angular Resolution survey of Protostellar Binary Systems", ApJ, 768, 110 (2013).

[3] Gutermuth, R., Myers, P., Megeath, S., Allen, L., Pipher, J., Muzerolle, J., Porras, A., Winston, E., and Fazio, G., "Spitzer Observations of NGC 1333: A Study of Structure and Evolution in a Nearby Embedded Cluster", ApJ, 674, 336 (2008).

[4] National Research Council, [Astronomy and Astrophysics in the New Millenium], National Academies Press, Washington DC (2000).

[5] Kouveliotou, C., Agol, E., Batalha, N., Bean, J., Bentz, M., Cornish, N., Dressler, A., Figueroa-Feliciano, E., Gaudi, S., Guyon, O., Hartmann, D., Niemack, M., Ozel, F., Reynolds, C., Roberge, A., Straughn, A., Sheth, K., Weinberg, D., and Zmuidzinas, J., "Enduring Quests - Daring Visions (NASA Astrophysics in the Next Three Decades)", NASA, http://go.nasa.gov/1gGVkZY (2014).

[6] Rinehart, S., Rizzo, M., Benford, D., Fixsen, D., Veach, T., Dhabal, A., Leisawitz, D., Mundy, L., Silverberg, R., Barry, R., Staguhn, J., Barclay, R., Mentzel, J., Griffin, M., Ade, P., Pascale, E., Klemencic, G., Savini, G., and Juanola-Parramon, R., "The Balloon Experimental Twin Telescope for Infrared Interferometry (BETTII): An Experiment for High Angular Resolution in the Far-Infrared," PASP, 126, 660 (2014).

[7] Rizzo, M., "BETTII: A pathfinder to high-angular resolution observations of star-forming regions in the far-infrared", Ph.D. Thesis. University of Maryland, College Park MD (2016).

[8] Rizzo, M., Mundy, L., Dhabal, A., Fixsen, D., Rinehart, S., Benford, R. Juanola-Parramon, D., Leisawitz, D., Silverberg, R., and Veach, T., "Far-infrared double-Fourier interferometers and their spectral sensitivity", PASP, 127, 1045 (2015).

[9] Dhabal, A., Rinehart, S., Rizzo, M., Mundy, L., Sampler, H., Juanola-Parramon, R., Veach, T., Fixsen, D., Vila, J., and Silverberg, R., "Optics alignment of a balloon-borne far-infrared interferometer BETTII," JATIS 3(2), 024002 (2017).

[10] Dhabal, A., Rinehart, S., Rizzo, M., and Mundy, L., "Optics of the Balloon Experimental Twin Telescope for Infrared Interferometry (BETTII): delay lines and alignment," Proc. SPIE, 9907, 0T (2016).

[11] Furst, S., Dow, T., Garrard, K., Sohn, A., Fixsen, D., Rinehart, S., Mentzell, E., Veach, T., Rizzo, M., and Dhabal, A., "Design and validation of the mounting structure for BETTII balloon based telescope with thin-walled optics." JATIS, 2, 024001 (2016).

[12] Veach, T., Rinehart, S., Mentzell, J, Silverberg, R., Fixsen, D., Rizzo, M., Dhabal, A., Gibbons, C., and Benford, J., "The Balloon Experimental Twin Telescope for Infrared Interferometry (BETTII): Optical Design", Proc. SPIE, 9143, 2H (2014).

[13] Chuss, D. T., Wollack, E.J, Moseley, S.H, and Novak, G., "Interferometric Polarization Control”, App. Opt., 45, 6107 (2006).

[14] Chuss, D. T., Wollack, E. J., Henry, R., Hui, H., Juarez, A. J., Krejny, M., Moseley, S. H., \& Novak, G., "Properties of a Variable-Delay Polarization Modulator", App. Opt., 51, 197 (2012).

[15] Benford, Dominic J., Staguhn, Johannes G., Ames, Troy J., Allen, Christine A., Chervenak, James A., Kennedy, Catherine R., Lefranc, Sebastien, Maher, Stephen F., Moseley, S. Harvey, Pajot, François, Rioux, Cyrille, Shafer, Richard A., Voellmer, George M., "First astronomical images with a multiplexed superconducting bolometer array", Proc. SPIE, 6275, 43 (2008).

[16] Allen, Christine A., Abrahams, John, Benford, Dominic J., Chervenak, James A., Chuss, David T., Staguhn, Johannes G., Miller, Timothy M., Moseley, S. Harvey, and Wollack, Edward J., "Far infrared through millimeter backshort-under-grid arrays", Proc. SPIE, 6275, 9 (2006).

[17] Battistelli, E. S., Amiri, M., Burger, B., Halpern, M., Knotek, S., Ellis, M., Gao, X., Kelly, D., MacIntosh, M., Irwin, K., and Reintsema, C., "Functional Description of Read-out Electronics for Time-Domain Multiplexed Bolometers for Millimeter and Sub-millimeter Astronomy,” J. Low. Temp. Phys., 151, 908 (2008). 
[18] Leach, R. W., "Multiple Readout CCD Controllers", Optical Astronomy from the Earth and Moon, 55, 113 (1994).

[19] Leach, R. W., “CCD Controllers”, New Developments in Array Technology and Applications, 167, 49 (1995).

[20] Benford, D. J., Fixsen, D. J., Rinehart, S. A., Rizzo, M., Maher, S. F., and Barry, R. K., "Precision Atitude Control for the BETTII Balloon-Borne Interferometer", Proc. SPIE 8444, 2P (2012).

[21] Rizzo, M., Rinehart, S., Alcorn, J., Barclay, R., Barry, R., Benford, D., Dhabal, A., Fixsen, D., Gore, A., JohnsonShapoval, S., Leisawitz, D., Maher, S., Mundy, L., Papageorgio, A., Pascale, E., Rau, A., Silverberg, R., Taraschi, P., Veach, T., and Weinreich, S., "Building an Interferometer at the Edge of Space: Pointing and Phase Control System for BETTII", Proc. SPIE 9143, 3H (2014).

[22] Vila Hernández de Lorenzo, J., Rinehart, S., Nehmetallah, G., Maher, S., Rizzo, M., Dhabal, A., Fixsen, D., Casalprim Torrse, M., Veach, T., and Esteves, F., "Flight performance of the attitude control system of the balloon experimental twin telescope for infrared interferometry (BETTII)," Proc. SPIE 10700, 213 (2018).

[23] Veach, T., et al., in prep (2018).

[24] Veach, T., Rinehart, S., Fixsen, D., Dhabal, A., Vila Hernández de Lorenzo, J., Maher, S., Rizzo, M., and Sharp, E., "Lessons learned from the investigation of an anomalous termination of BETTII," Proc. SPIE 10700, 75 (2018).

[25] Nordgren, T. \& Hajian, A., "A New Multichannel Fourier Transform Spectrometer," in Precise Stellar Radial Velocities, ASP Conf. 185, IAU Colloquium 170, 36 (1999).

[26] Huang, D, Swanson, EA, Lin, CP, Schuman, JS, Stinson, WG, Chang, W, Hee, MR, Flotte, T, et al., "Optical coherence tomography". Science 254 (5035): 1178-81 (1991).

[27] Dhabal, A., Rinehart, S., Mentzell, E., Fixsen, D., Rizzo, M., Veach, T., Juanola-Parramon, R., and Vila Hernández de Lorenzo, “A Dispersive backend design for the double-fourier interferometer BETTII," Proc. SPIE 10701, 35 (2018). 